\title{
Reduced expression of PD-L1 in autoimmune thyroiditis attenuate trophoblast invasion through ERK/MMP pathway
}

Mengya Chen, Nduwimana Gilbert and Haixia Liu*

\begin{abstract}
Background: Autoimmune thyroiditis (AIT) with euthyroid is associated with miscarriage. But the exact mechanism remains unclear. Studies have shown that the programmed cell death-1 (PD-1)/programmed cell death -ligand 1 (PD-L1) pathway is essential for normal pregnancy. However, the expression of PD-L1 in gestational trophoblasts in mice with autoimmune thyroiditis and the mechanisms leading to miscarriage have not been fully investigated.

Methods: Immunofluorescence and Western blot were used to detect the expression of PD-L1, p-ERK, MMP-2 and MMP-9 in embryonic trophoblast cells of pregnant mice with AIT. The expression of PD-L1 in HTR-8/SVneo cells were silenced, and the expression of PD-L1, MMP-2, MMP-9, ERK and p-ERK1/2 was detected by Western blot analyses and immunofluorescence assays. Invasive assays were performed in PD-L1 silenced HTR-8/SVneo cells using a Transwell chamber.
\end{abstract}

Results: Compared with normal pregnancy, the expression of PD-L1, ERK, p-ERK, MMP-2 and MMP-9 in embryonic trophoblast cells was significantly lower in pregnant mice with AIT. Compared with the negative control (NC) group (cells transfected with negative control siRNA), phosphorylation of MMP-2, MMP-9 and P-ERK1/2 proteins was significantly reduced in HTR-8/SVneo cells transfected with PD-L1 siRNA, and the number of cells penetrating the membrane was reduced.

Conclusion: AIT inhibits ERK/MMP-2 and MMP-9 pathways through PD-L1 reduction, attenuates embryonic trophoblast invasion and ultimalely induces miscarriage ultimately.

Keywords: PD-1/PD-L1, AIT, Placenta, Invasion, ERK1/2

\section{Introduction}

A lot of evidence suggests that the most common and serious adverse pregnancy outcomes caused by AIT with euthyroid are miscarriages, especially repeated abortions [1, 2]. However, the specific mechanism is still unclear. The invasive behavior of extravillous trophoblast cells (EVT) is an essential element in the formation and development of placenta and the successful completion of pregnancy [3, 4]. EVT cells originate from cytotrophoblastic (CTB) cells and then invade the upper third of the aponeurosis and

\footnotetext{
* Correspondence: dllhxsci@163.com

Department of Endocrinology and Metabolism, The Second Affiliated Hospital of Dalian Medical University, 467 Zhongshan Road, Dalian 116027, Liaoning, China
}

myometrium while reconstructing the associated spiral arteries [5]. The invasive ability of EVT cells is strictly regulated by various growth and regulatory factors in the endometrial microenvironment of the entire pregnancy, mainly the decidua [6]. This regulation is carried out in a strict space and time mode, and the destruction of such regulations may lead to adverse consequences $[3,7]$.

$\mathrm{PD}-1 / \mathrm{PD}-\mathrm{L}$ signaling pathway is a negative costimulatory pathway found in recent years [8]. PD-1 is mainly expressed on the surface of activated T cells, while PD-L1 is mainly expressed in antigen-presenting cells and immunologically exempt sites (such as placenta). Holets et al. [9] found that PD-L1 is expressed on all trophoblast cells in human placenta. The PD-L1 protein of tumor cells 
binds to the PD-1 receptor on $\mathrm{T}$ cells, $\mathrm{T}$ cells cannot recognize tumor cells, and tumor cells open immune escape, which gives the tumor cells strong invasiveness [10, 11]. Studies have shown that extracellular signal-regulated kinase (ERK) signaling is mediated by PD-L1, which activates ERK signaling through PD-L1 to confer tumor invasiveness $[12,13]$. The invasion of trophoblast cells is similar to that of tumor cells [3]. In addition, ERK1/2 phosphorylation has been shown to be involved in the regulation of MMP-2/- 9 expression [14]. The trophoblast invasion ability is closely related to the expression of MMP-2/-9, because MMP-2/- 9 is the main enzyme that degrades the basement membrane, and the extracellular degradation matrix (ECM) and basement membrane are the initial processes to promote cell invasion $[15,16]$. Studies have shown that the ERK signal transduction pathway regulates the invasion of trophoblasts by activating cell adhesion molecules and myosin, up-regulating the expression of MMP-9 [17]. The study found that there were MMP-2 and MMP-9 synthesis in the decidual cells of the pseudopregnant mice on days 6-8 [18]. In vitro experiments showed that the mRNA and protein of MMP-2 and MMP-9 were elevated in trophoblast cells in early pregnancy [19]. Based on the above evidence, we hypothesized that AIT due to the decrease of PD-L1 content in embryonic trophoblast cells, which then affects the expression of MMP-2/-9 through ERK signaling pathway, and reduce the invasiveness of trophoblast cells to induce abortion.

\section{Materials and methods Immunisation protocols}

AIT modelling of CBA/J mice with thyroglobulin using previously reported methods [20]. In order to induce autoimmune thyroiditis, $\mathrm{CBA} / \mathrm{J}$ mice were first immunised with mTg $(100 \mu \mathrm{g} /$ mouse $)$ in complete Freund's adjuvant (Sigma, F5881) at 5 weeks of age and were then challenged with $\mathrm{mTg}(100 \mu \mathrm{g} / \mathrm{mouse})$ in incomplete Freund's adjuvant (Sigma, F5506) at 7 weeks of age. The same dose of phosphate buffered saline (PBS) instead of mTg was used to immunize the control group mice, and the other methods were the same as those of the mTg group. CBA/J (Beijing HFK Bioscience Co) AIT pregnant mice were sacrificed by cervical dislocation at 13.5th day of gestation, and placental tissue was isolated by laparotomy.

\section{Thyroid function tests}

$\mathrm{TT}_{4}$ (Signalway Antibody, EK18886) TSH (Elabscience Biotechnology Co, Ltd) and anti-Tg antibody (Elabscience Biotechnology Co, Ltd) were determined by ELISA. All samples were measured twice, and specific experimental steps were performed in accordance with the kit specifications.

\section{Immunohistochemistry}

The embedded placental tissue sections were treated using standard immunohistochemical techniques. Antibodies used in immunohistochemistry experiments included an anti-PD-L1 Ab (1:800 dilution; Proteintech, 66,248-1-Ig), anti- p-ERK Ab (1:300 dilution; CST, Thr202/Tyr204, mAb \#4370), anti-MMP-2 (1:200 dilution; Proteintech, 10,373-2-AP) and anti- MMP-9 Ab (1: 200 dilution; Proteintech, 10,375-2-AP). Immunoreactivity was evaluated independently by two investigators who were blinded to experimental protocol according to the intensity and extent of staining. Immunohistochemistry images were obtained with confocal microscopy (Leica DM4000B) and immunoreactivity using at least 3 random microscopes field of view. The experimental results were analyzed using Image-Pro Plus software (version 6.0; Media Cybernetics).

\section{Cell culture}

HTR-8/SVneo cells (EK-Bioscie, Shanghai, CHN) were cultured in RPMI-1640 medium (HyClone, US) containing $10 \%$ fetal bovine serum (FBS, AusGeneX, AUS), 1\% penicillin-Streptococcus bismuth antibody (HyClone, US), and incubated under $5 \% \mathrm{CO}_{2}$ at $37^{\circ} \mathrm{C}$.

\section{siRNA transfection and gene silencing}

HTR-8/SVneo cells were classified into a control group, a small interfering negative control (NC) group (cells transfected with negative control siRNA) and two different small interfering RNAs groups (cells transfected with PDL1 siRNA1 and siRNA2). The PD-L1 siRNAs and negative control siRNA used in the present study were provided by Shanghai GenePharma Co., Ltd. (Shanghai, China). The transfection of cells was performed with Lipofectamin 2000 (Invitrogen, Carlsbad, CA) for a final concentration of 50 nM siRNA/well, according to the manufacturer's protocol. Cells were harvested $48 \mathrm{~h}$ post-transfection for further analyses. The inhibition efficiency was identified by western blot. The sequence of PD-L1 siRNA1: sense: 5 '-GCC GAA GUC AUC UGG ACA ATT-3', antisense: 5'-UUG UCC AGA UGA CUU CGG CTT-3'. The sequence of PD-L1 siRNA2: sense: 5'-GAA GCA AAG UGA UAC ACA UTT-3', antisense: $5^{\prime}$-AUG UGU AUC ACU UUG CUU CTT-3'. The sequence of NC siRNA: sense: $5^{\prime}$ UUC UCC GAA CGU GUC ACG UTT-3', antisense: 5' ACG UGA CAC GUU CGG AGA ATT-3'.

\section{Western blot analysis}

Proteins were cleaved from CBA/J mouse placenta and HTR-8/SVneo cells for Western blot analysis. Standard Western blotting techniques were used to determine protein expression; $10 \mu \mathrm{g}$ of protein was separated by $10 \%$ SDS-PAGE, transferred to a polyvinylidene fluoride membrane, and treated with anti-PD-L1 (1:2000 dilution; 
Proteintech, 66,248-1-Ig), anti- p-ERK Ab (1:2000 dilution; Cell Signaling, Thr202/Tyr204, mAb \#4370), antiMMP2 (1:2000 dilution; Proteintech, 10,373-2-AP), antiMMP9 Ab (1:2000 dilution; Proteintech, 10,375-2-AP) and anti-GAPDH (1:2000 dilution; Cell Signaling) Ab overnight at $4{ }^{\circ} \mathrm{C}$ in a refrigerator and then with the second antibody (1:2000 dilution; Cell Signaling) it was incubated for $90 \mathrm{~min}$. The signal was detected in an enhanced ECL system (GE/Amersham).

\section{Immunofluorescence staining and analysis}

The cells cultured on the cell slides were washed three times with phosphate buffered saline (PBS), fixed with PBS containing $4 \%$ paraformaldehyde for $10 \mathrm{~min}$, and then incubated in Triton X-100-containing PBS for room temperature permeabilization. After $10 \mathrm{~min}$, the cells were washed with PBS, we added PD-L1 (1:200 dilution; Proteintech, 66,248-1-Ig), MMP-2 (1:200 dilution; Proteintech, 10, 373-2-AP), MMP-9 (1:200 dilution; Proteintech, 10,375-2AP), ERK1/2 (1:200 dilution; Cell Signaling) and phosphorylated ERK (1:200 dilution; Cell Signaling, Thr202/Tyr204, mAb \#4370) primary antibody (1:200, Abcam) overnight at $4{ }^{\circ} \mathrm{C}$, anti-rabbit fluorescent secondary antibody (1:50, Thermo) incubate at room temperature for $1 \mathrm{~h}, 1 \mu \mathrm{g} / \mathrm{mL}$ DAPI (Roche diagnostics) at $37^{\circ} \mathrm{C}$ for $15 \mathrm{~min}$, remove excess water, add fluorescent sealing solution, cover the coverslip, and observe under a laser confocal microscope (Olympus BX63).

\section{Cell invasion assay}

HTR-8/SVneo cells in the control group, NC group, siRNA1 group and siRNA2 group were first incubated for $48 \mathrm{~h}$. Cell invasion assays were performed using a transwell chamber (Corning) pre-coated with Matrigel (BD, USA). RPMI-1640 medium containing 15\% FBS was added to $600 \mathrm{ml}$ of the lower chamber. The cells were then washed with PBS and suspended in MEM. Two hundred milliliter of cell suspension $\left(1 \times 10^{5}\right.$ cells/ $\mathrm{ml})$ was added to the upper chamber. After incubation at $37^{\circ} \mathrm{C}$ for $36 \mathrm{~h}$, the upper cells of the non-invasive membrane were wiped with a cotton swab. The filter was fixed in methanol and stained with $4 \mathrm{~g} / \mathrm{L}$ crystal violet. The number of invading cells whose nuclei were stained purple was counted under microscope.

\section{Statistical analyses}

Density values of proteins Bands obtained by Western Blot were detected by Image-pro Plus 6 software. All data were statistically analyzed using SPSS 20.0 or Graph Pad Prism6 software. The difference was statistically significant, $P<0.05$.

\section{Results}

Information about the AIT pregnant mice

Building a murine fetal loss model of isolated positive maternal $\operatorname{TgAb}$ The first step of the experiment was to construct a TgAb-positive aborted animal model. No significant difference was found in the serum $\mathrm{TT}_{4}$ and TSH level after immunization with mTg (Table 1). The serum $\mathrm{TgAb}$ level in the mTg group was significantly higher than that in the con group (Table 2, $P<0.05$ ). Embryo implantation rates were reduced in $\mathrm{mTg}$ group compared to control group $(94.63 \%$ vs. $53.21 \% ; P<0.05$. Fetal resorption rates were increased in $\mathrm{mTg}$ group compared to the control group (Fig. 1c) and the volume of embryos in the mTg group was significantly smaller than that of the control groups, as shown in Fig. 1a-b. This may be due to conditions such as ischemia, hemorrhage, and necrosis in the embryo.

\section{PD-L1, phosphorylated ERK, MMP-2 and MMP-9 expression in embryonic trophoblast cells of AIT pregnant mice}

The results of immunohistochemistry showed that, compared with the control group, the amount of PDL1 (Fig. 2a) in the embryonic trophoblast cells of pregnant mice with AIT was significantly decreased. The amount of phosphorylated ERK (Fig. 2b), MMP-2 (Fig. 2c) and MMP-9 (Fig. 2d) was also significantly reduced.

To further compare the expressions of PD-L1 in the placenta, we used Western Blot to detect the PDL1, t-ERK, p-ERK, MMP-2 and MMP-9 expressions in the placental tissue proteins of normal pregnant mice and AIT pregnant mice. The results showed that the PD-L1, p-ERK, MMP-2 and MMP-9 expressions in placental tissue proteins of AIT pregnant mice was significantly reduced (Fig. 2e).

\section{Silencing of PD-L1 reduced the levels of phosphorylated ERK, MMP-2 and MMP-9 of HTR-8/SVneo cells}

To assess whether phosphorylated ERK, MMP-2 and MMP-9 changes were caused by a decrease in PD-L1, we used siRNAs targeting PD-L1 to inhibit the PD-L1 expression. Compared with NC cells, the expression of PD-L1 (Fig. 3a), phosphorylated ERK (Fig. 3b), MMP-2 (Fig. 3c) and MMP-9 (Fig. 3d) was significantly decreased in cells transfected with siRNAs to PD-L1, and confirmed by the cellular immunofluorescence analysis.

Table 1 The comparison of the serum levels of TSH and T4 $(\bar{x}$
\begin{tabular}{lll} 
\pm S.E.M $)$ & \\
\hline Groups & TSH $(\mathrm{mlU} / \mathrm{L})$ & $\Pi_{4}(\mu \mathrm{g} / \mathrm{dL})$ \\
\hline $\operatorname{Con}(n=10)$ & $0.3306 \pm 0.03495$ & $3.907 \pm 0.4839$ \\
$\mathrm{mTg}(n=10)$ & $0.3165 \pm 0.04059$ & $3.595 \pm 0.3682$ \\
\hline
\end{tabular}


Table 2 The comparison of the serum levels of $\operatorname{TgAb}(\bar{x} \pm$ S.E.M)

\begin{tabular}{ll}
\hline Groups & $\operatorname{TgAb}(\mu \mathrm{g} / \mathrm{dL})$ \\
\hline $\operatorname{Con}(n=10)$ & $1.805 \pm 0.030$ \\
$\mathrm{mTg}(n=10)$ & $37.25 \pm 2.800$ \\
\hline
\end{tabular}

Western blot detection results were the same as the above (Fig. 3e). These results indicate that the PD-1/PDL1 signaling pathway is involved in the regulation of MMP-2 and MMP-9 expression and secretion in HTR$8 / \mathrm{SVneo}$ cells. The mitogen-activated protein kinase (MAPK) cascade is an important pathway regulating MMP-2 or MMP-9 expression and can respond to extracellular stimuli. Preferentially activates the extracellular signal-regulated kinase-1/2 (ERK1/2) signaling pathway. In HTR-8/SVneo cells transfected with PD-L1 siRNA, ERK1 phosphorylation at $44 \mathrm{kDa}$ (Thr202/Tyr204) increased significantly. However, there was no significant change in total ERK. These results indicate that the in vitro invasion of embryonic trophoblast cells may be mediated by MMP-2 and MMP-9 expression regulated by the MAPK/ERK cascade.
Silencing of PD-L1 decresed the invasion ability of HTR-8/ SVneo cells

To evaluate the biological role of PD-L1 in the trophoblast invasion of the placenta, HTR-8/SVneo cells were transfected with PD-L1 siRNAs (siRNA1 and siRNA2) to knock down PD-L1 for use in subsequent studies and used for subsequent studies. PD-L1 expression was significantly reduced in HTR-8/SVneo cells transfected with siRNAs targeting PD-L1, as confirmed by cellular immunofluorescence analysis (Fig. 2a) and Western blot analysis (Fig. 2e). To investigate the effect of PD-L1 on HTR-8/SVneo cell invasion, a Transwell chamber with a Matrigel-coated filter was used. Then $200 \mathrm{ml}$ different HTR-8/SVneo cells suspension were added into the upper chamber. MEM with 15\% FBS was added into the lower chamber. Following incubation for 36 $\mathrm{h}$, the invasive cells were stained with purple crystal and recorded under microscope. We found that compared with the NC group cells, HTR-8/SVneo cells transfected with siRNAs targeting PD-L1 had reduced invasion ability (Fig. 4).

\section{Discussion}

The mechanism of abortion caused by AIT has not been unanimously determined. So far, whether the PD-L1

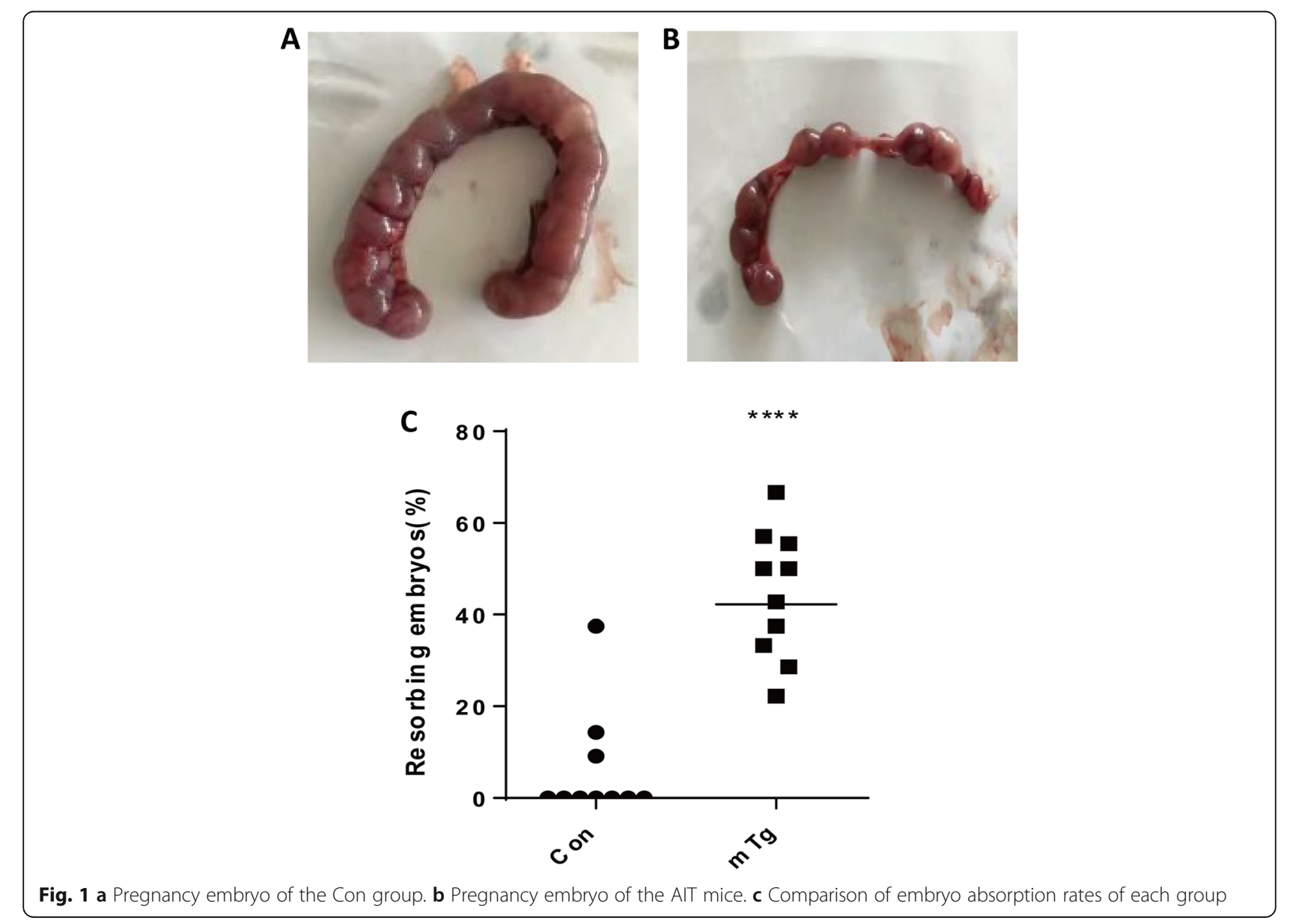




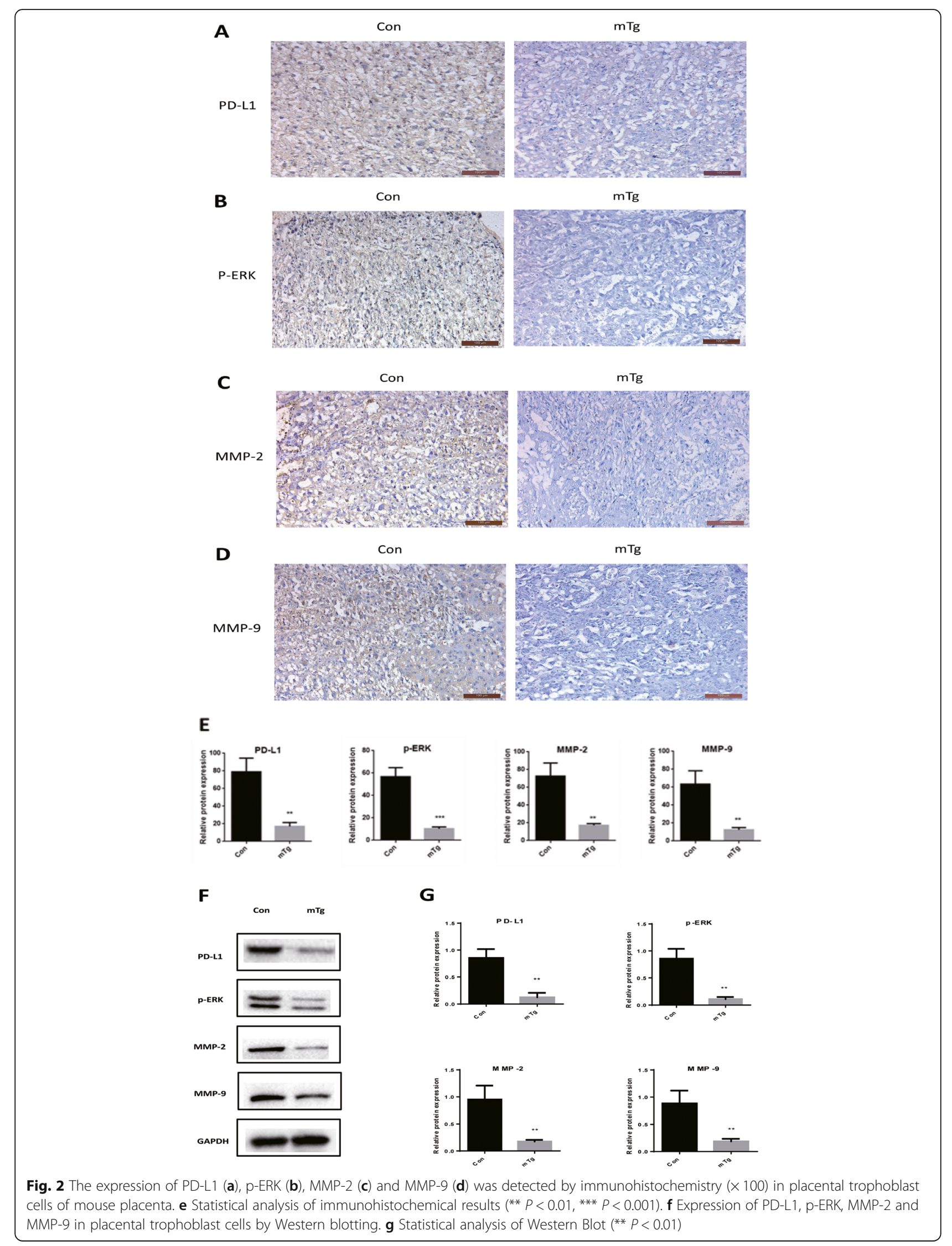



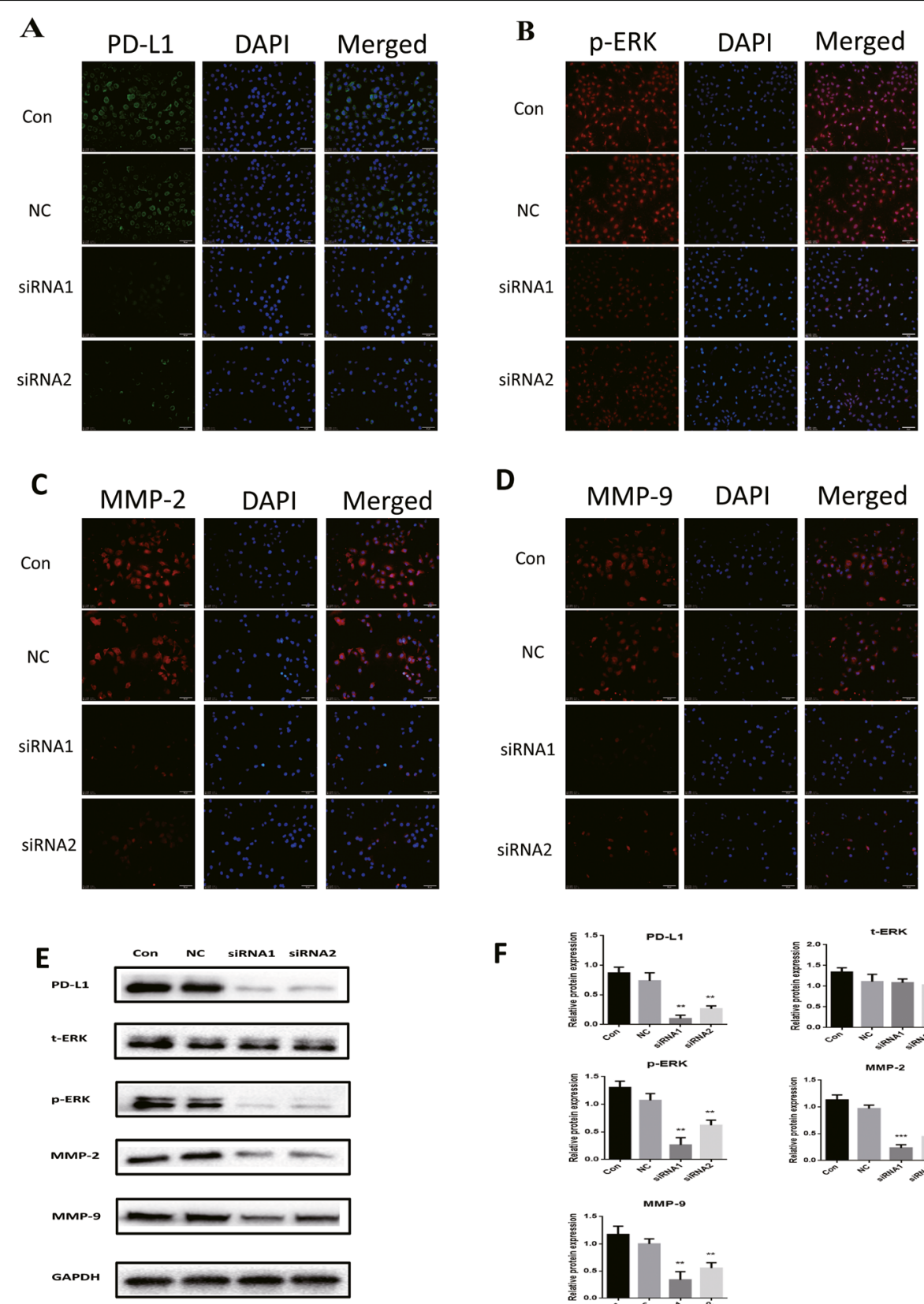

$\mathbf{F}$
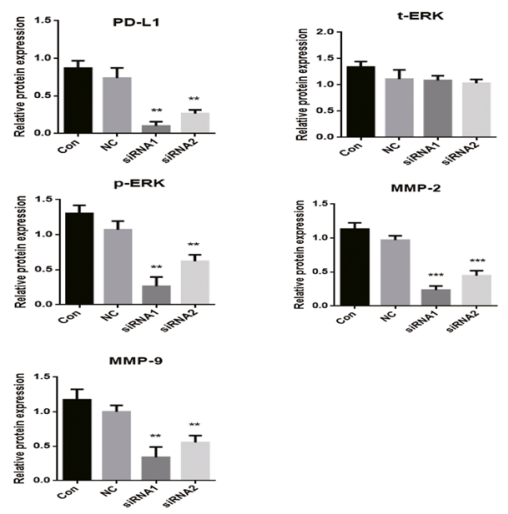

Fig. 3 Effective knockdown of PD-L1 by siRNA in HTR-8/SVneo cells. HTR-8/SVneo cells were pre-incubated with PD-L1 siRNA for 48 h, and the levels of PD-L1 (a), p-ERK (b), MMP-2 (c) and MMP-9 (d) in NC and siRNA groups were detected by immunofluorescence; $\mathbf{e}$ The protein expression of PD-L1, MMP-2, MMP-9, ERK1/2 and PERK1/2 were evaluated by western blot in transfected cells. $\mathbf{f}$ Statistical analysis of the western blot results $\left(* * P<0.01,{ }^{* * *} P<0.001\right)$

expression in placental trophoblast cells causes the invasion function of trophoblast cells is unknown. In recent years, immune checkpoints have played an important role in the process of cancer research. Among them, PD-1 is one of the most characteristic checkpoint proteins. PD-1 and its ligand PD-L1 are key regulators of T cell immune response and peripheral tolerance induction $[21,22]$. The interaction between PD-1 and PD-L1 can result in inhibition of $\mathrm{T}$ cells, allowing PD-L1 expressing cancer cells to evade PD-1 positive immune cells [23]. Evading immune surveillance and preventing subsequent rejection of the host immune system are the basis for maintaining uterine embryo and fetal development as they express allogeneic paternal antigens [24]. At the maternal-fetal interface, PD-1 is expressed on activated decidual $\mathrm{T}$ cells [25], and during pregnancy, PD- 

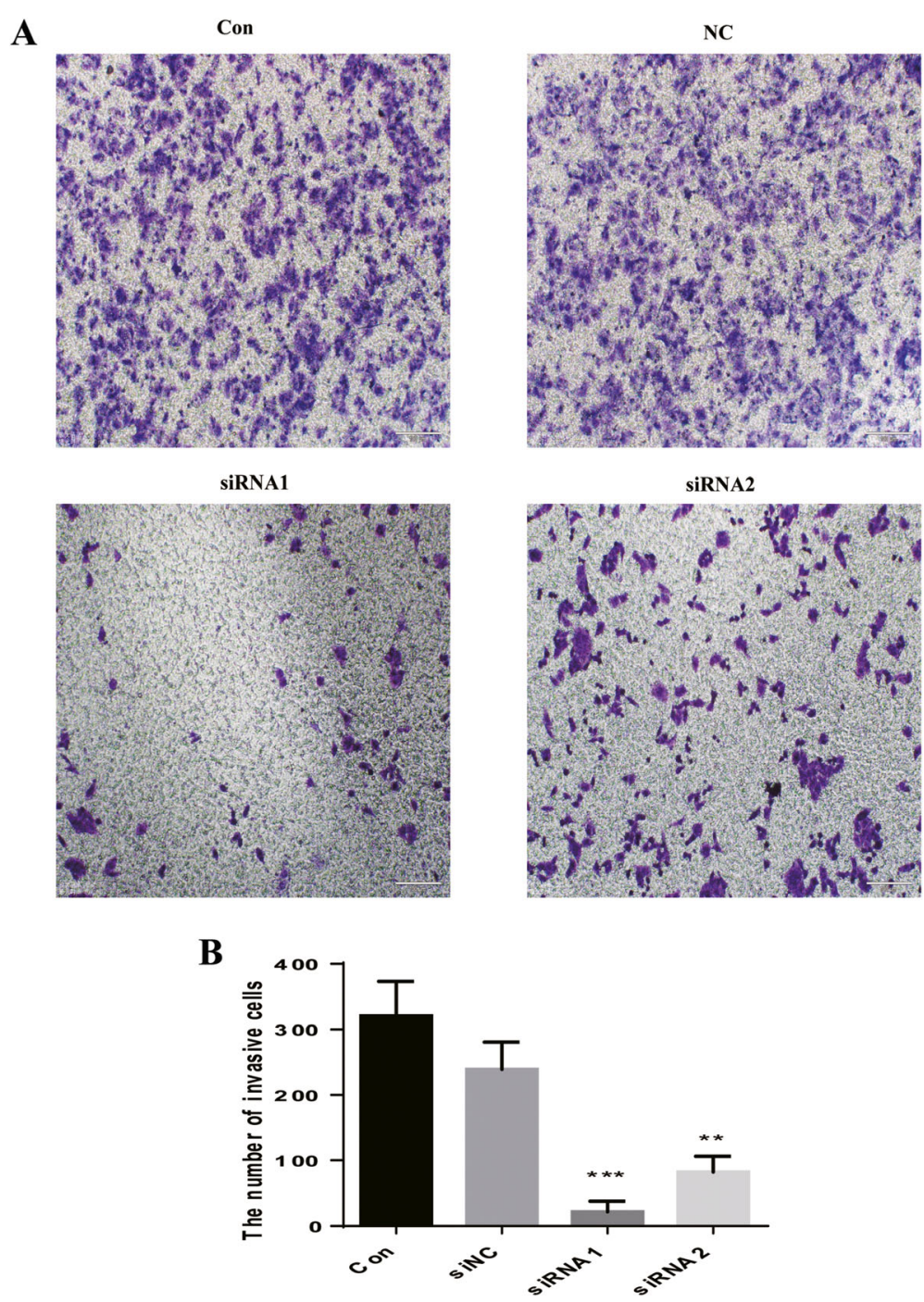

Fig. 4 Effect of PD-L1 knockdown on cell invasion in HTR-8/SVneo cells. Parental or HTR-8/SVneo cells transfected with negative control siRNA (NC) or siRNAs (siRNA1 siRNA2) targeting PD-L1 for $48 \mathrm{~h}$ were seeded in modified transwell chamber with Matrigel-coated membrane, and after another $24 \mathrm{~h}$, invasive cells that moved through the Matrigel membrane were stained and counted under a microscope $(\times 100)$. a Showed the nuclei of the invasive cells. $\mathbf{b}$ Showed statistical analysis of the number of invasive cells. Data was shown as means \pm SD from five fields. ${ }^{* *} P<$ 0.01 versus the NC group

L1 is expressed in embryonic trophoblast cells [26]. In non-gestational endometrium, the density of $\mathrm{PD}-1^{+} / \mathrm{CD}^{+}$ lymphocytes was lower than that in the first trimester placental sites, suggesting that PD-L1 expressing trophoblast may exploit PD-1/PD-L1 mediated immune suppression in normal gestation. In fact, it has been reported that pregnant mice treated with anti-PD-L1 blocking antibody lose their embryos [27] and a deficiency in PD-L1 has been associated with an increased frequency of fetal resorption and decreased fetal survival [28]. Survival of the developing embryo and fetus requires immune tolerance by inactivating the maternal immune system at the placentalmaternal interface, which is thought to be accomplished by trophoblast [24]. Invasion of extravillous trophoblasts plays an important role in embryo implantation and placental formation. If this invasion is inhibited, it will cause abortion due to placental dysfunction.

In this study, we have confirmed that PD-L1 levels are significantly reduced in placental trophoblast cells of pregnant mice with autoimmune thyroiditis. To verify whether the inhibition of the trophoblast cells was affected by inhibition of the PD-L1/PD-L1 signaling pathway, we downregulated PD-L1 expression in HTR-8/SVneo cells, which indicates that abnormally low expression of PD-L1 in HTR-8/SVneo cells results in a significant decrease in the invasive ability of the cells.

The invasion of trophoblast cells is a very complex process that may be affected by many molecules that 
can affect cell growth, adhesion, differentiation, and degradation of extracellular matrix (ECM), among which, the proteolytic degradation of ECM plays an important role in the process of trophoblast invasion into the endometrium [29]. Matrix metalloproteinases (MMPs) are a key family of proteolytic enzymes involved in trophoblast invasion. Studies have shown that MMP-2 is one of the key enzymes for the degradation of type IV collagen during cell invasion, MMP-9 is identified as trophoblast derived MMP [30]. To investigate how the PD-1/PD-L1 signaling pathway affects cell invasion, we examined the expression levels of MMP-2 and MMP-9 in PD-L1 siRNA-transfected cells. The results showed that the expression of MMP-2 and MMP-9 was down-regulated in cells knocked down by PD-L1. This suggests that attenuation of the PD-1/PD-L1 signaling pathway in the placenta of pregnant mice with AIT may inhibit trophoblast invasion by decreasing the expression of MMP-2 and MMP-9. Previous studies have demonstrated that MMP activation is caused by ERK1/2 phosphorylation [31]. Therefore, we examined the expression of phosphorylated ERK1/2 and total ERK1/2. The results showed that there was no difference in ERK1/2 protein levels in the HTR-8/SVneo cells transfected with PD-L1 siRNA compared with the NC group, but the phosphorylated ERK1/2 levels decreased significantly, indicating that the weakening of the PD-1/PD-L1 signaling pathway leading to phosphorylation of ERK1/2 is reduced. In addition, the results also showed that the expression of MMP-2 and MMP-9 was decreased in HTR-8/SVneo cells transfected with PDL1 siRNA. Thus, we infer that, in the placenta of pregnant mice with autoimmune thyroiditis, attenuation of the PD1/PD-L1 signaling pathway may inhibit p-ERK1/2 signaling and its downstream effects on MMP-2 and MMP-9.

In summary, our results suggest that decreased invasiveness of embryonic trophoblasts in autoimmune thyroiditis is associated with down-regulation of PD-1/PDL1 signaling pathway and inhibition of MMP-2 and MMP-9 expression. However, studies of mouse placenta and in vitro human trophoblast cells do not fully mimic the normal placental uterus. Therefore, the exploration of these findings requires further research.

\section{Abbreviations}

AIT: Autoimmune thyroiditis; CTB: Cytotrophoblastic; ERK: Extracellular signalregulated kinase; EVT: Extravillous trophoblast cells; FBS: Fetal bovine serum; HE: Hematoxylin-eosin; MAPK: Mitogen-activated protein kinase; MMP: Matrix metalloproteinases; PD-1: Programmed cell death-1; PD-L1: Programmed cell death -ligand 1; RPMI: Roswell Park Memorial Institute

\section{Acknowledgments}

We are grateful to the Second Affiliated Hospital of Dalian Medical University for their support of this experiment.

\section{Authors' contributions}

MC created the original idea and structure for the paper, contributed to study design, data interpretation and manuscript editing; HL made substantial contributions to conception and design and have been involved in drafting the manuscript and revising it critically for important intellectual content. NG contributed to manuscript writing in English and editorial revisions. All authors read and approved the final manuscript.

\section{Funding}

The study was supported by the National Natural Science Foundation of China (Grant 81600605); Scientific and Technological Innovation Project of Dalian Science and Technology Bureau (Grant 2018J13SN094), and Clinical Capability Construction Project for Liaoning Provincial Hospitals, Health Care Commission, Liaoning Province Government of China (Grant LNCCC-D232015).

The effect of PD-1/PD-L1 pathway on autoimmune thyroiditis and immune mechanism in Liaoning Natural Science Foundation Project in 2019 (No. 2019-MS-083).

\section{Availability of data and materials}

All data generated or analysed during this study are included in this published article.

\section{Ethics approval and consent to participate}

Experimental protocols using mice were approved by the Experimental Animal Ethics Committee of Dalian Medical University (approval number: L20160242), and all of the animals were treated in accordance with the Institutional Guidelines for Experiments Using Animals.

\section{Consent for publication}

Not applicable.

\section{Competing interests}

The authors declare that they have no competing interests.

Received: 3 July 2019 Accepted: 16 October 2019

Published online: 27 October 2019

\section{References}

1. Stagnaro-Green A, Glinoer D. Thyroid autoimmunity and the risk of miscarriage. Best Pract Res Clin Endocrinol Metab. 2004;18:167-81.

2. Prummel MF, Wiersinga WM. Thyroid autoimmunity and miscarriage. Eur J Endocrinol. 2004;150:751-5.

3. Anin SA, Vince G, Quenby S. Trophoblast invasion. Hum Fertil (Camb). 2004; 7:169-74.

4. Pijnenborg R, Bland JM, Robertson WB, Brosens I. Uteroplacental arterial changes related to interstitial trophoblast migration in early human pregnancy. Placenta. 1983;4:397-413.

5. Pijnenborg R, Dixon G, Robertson WB, Brosens I. Trophoblastic invasion of human decidua from 8 to 18 weeks of pregnancy. Placenta. 1980;1:3-19.

6. Graham CH, Lala PK. Mechanism of control of trophoblast invasion in situ. J Cell Physiol. 1991;148:228-34.

7. Zhu JY, Pang ZJ, Yu YH. Regulation of trophoblast invasion: the role of matrix metalloproteinases. Rev Obstet Gynecol. 2012;5:e137-43.

8. Brown JA, Dorfman DM, Ma FR, Sullivan EL, Munoz O, Wood CR, Greenfield EA, Freeman GJ. Blockade of programmed death-1 ligands on dendritic cells enhances T cell activation and cytokine production. J Immunol. 2003;170:1257-66.

9. Holets LM, Hunt JS, Petroff MG. Trophoblast CD274 (B7-H1) is differentially expressed across gestation: influence of oxygen concentration. Biol Reprod. 2006;74:352-8.

10. Nicolazzo C, Gradilone A, Carpino G, Gazzaniga P, Raimondi C. Molecular characterization of circulating tumor cells to study Cancer Immunoevasion. Methods Mol Biol. 2019;1884:247-58.

11. Morimoto Y, Kishida T, Kotani SI, Takayama K, Mazda O. Interferon-beta signal may up-regulate PD-L1 expression through IRF9-dependent and independent pathways in lung cancer cells. Biochem Biophys Res Commun. 2018:507:330-6

12. Lin PL, Wu TC, Wu DW, Wang L, Chen CY, Lee $H$. An increase in BAG-1 by PD-L1 confers resistance to tyrosine kinase inhibitor in non-small cell lung cancer via persistent activation of ERK signalling. Eur J Cancer. 2017:85:95-105.

13. Lin HY, Chin YT, Nana AW, Shih YJ, Lai HY, Tang HY, Leinung M, Mousa SA, Davis PJ. Actions of I-thyroxine and Nano-diamino-tetrac (Nanotetrac) on PD-L1 in cancer cells. Steroids. 2016;114:59-67. 
14. Sun X, Lin L, Chen Y, Liu T, Liu R, Wang Z, Mou K, Xu J, Li B, Song H. Nitidine chloride inhibits ovarian cancer cell migration and invasion by suppressing MMP2/9 production via the ERK signaling pathway. Mol Med Rep. 2016;13:3161-8.

15. Spallarossa P, Altieri P, Garibaldi S, Ghigliotti G, Barisione C, Manca V, Fabbi P, Ballestrero A, Brunelli C, Barsotti A. Matrix metalloproteinase-2 and -9 are induced differently by doxorubicin in H9c2 cells: the role of MAP kinases and NAD(P)H oxidase. Cardiovasc Res. 2006;69:736-45.

16. Gweon EJ, Kim SJ. Resveratrol induces MMP-9 and cell migration via the p38 kinase and PI-3K pathways in HT1080 human fibrosarcoma cells. Oncol Rep. 2013;29:826-34.

17. Pollheimer J, Knofler M. Signalling pathways regulating the invasive differentiation of human trophoblasts: a review. Placenta. 2005;26(Suppl A):S21-30.

18. McCawley $\sqcup$, Matrisian LM. Matrix metalloproteinases: they're not just for matrix anymore! Curr Opin Cell Biol. 2001;13:534-40.

19. Isaka K, Usuda S, Ito H, Sagawa Y, Nakamura H, Nishi H, Suzuki Y, Li YF, Takayama M. Expression and activity of matrix metalloproteinase 2 and 9 in human trophoblasts. Placenta. 2003;24:53-64.

20. Imaizumi M, Pritsker A, Kita M, Ahmad L, Unger P, Davies T. Pregnancy and murine thyroiditis: thyroglobulin immunization leads to fetal loss in specific allogeneic pregnancies. Endocrinology. 2001;142:823-9.

21. Francisco LM, Sage PT, Sharpe AH. The PD-1 pathway in tolerance and autoimmunity. Immunol Rev. 2010;236:219-42.

22. Gianchecchi E, Delfino DV, Fierabracci A. Recent insights into the role of the PD-1/PD-L1 pathway in immunological tolerance and autoimmunity. Autoimmun Rev. 2013;12:1091-100.

23. Chin YT, Wei PL, Ho Y, Nana AW, Changou CA, Chen YR, Yang YS, Hsieh MT, Hercbergs A, Davis PJ, et al. Thyroxine inhibits resveratrol-caused apoptosis by PD-L1 in ovarian cancer cells. Endocr Relat Cancer. 2018;25:533-45.

24. Veras E, Kurman RJ, Wang TL, Shih IM. PD-L1 expression in human placentas and gestational trophoblastic diseases. Int J Gynecol Pathol. 2017;36:146-53.

25. Xu YY, Wang SC, Li DJ, Du MR. Co-signaling molecules in maternal-fetal immunity. Trends Mol Med. 2017;23:46-58.

26. Wang $S$, Zhu X, XU Y, Zhang D, Li Y, Tao Y, Piao H, Li D, Du M. Programmed cell death-1 (PD-1) and T-cell immunoglobulin mucin-3 (Tim-3) regulate CD4+ T cells to induce type 2 helper T cell (Th2) bias at the maternal-fetal interface. Hum Reprod. 2016;31:700-11.

27. D'Addio F, Riella LV, Mfarrej BG, Chabtini L, Adams LT, Yeung M, Yagita $H$, Azuma M, Sayegh MH, Guleria I. The link between the PDL1 costimulatory pathway and Th17 in fetomaternal tolerance. J Immunol. 2011;187:4530-41.

28. Habicht A, Dada S, Jurewicz M, Fife BT, Yagita H, Azuma M, Sayegh MH, Guleria I. A link between PDL1 and T regulatory cells in fetomaternal tolerance. J Immunol. 2007;179:5211-9.

29. Saito S, Nakashima A. A review of the mechanism for poor placentation in early-onset preeclampsia: the role of autophagy in trophoblast invasion and vascular remodeling. J Reprod Immunol. 2014;101-102:80-8.

30. Jia RZ, Rui C, Li JY, Cui XW, Wang X. CDX1 restricts the invasion of HTR-8/SVneo trophoblast cells by inhibiting MMP-9 expression. Placenta. 2014;35:450-4.

31. Zou J, Xu L, Ju Y, Zhang P, Wang Y, Zhang B. Cholesterol depletion induces ANTXR2-dependent activation of MMP-2 via ERK1/2 phosphorylation in neuroglioma U251 cell. Biochem Biophys Res Commun. 2014;452:186-90.

\section{Publisher's Note}

Springer Nature remains neutral with regard to jurisdictional claims in published maps and institutional affiliations.

Ready to submit your research? Choose BMC and benefit from:
- fast, convenient online submission
- thorough peer review by experienced researchers in your field
- rapid publication on acceptance
- support for research data, including large and complex data types
- gold Open Access which fosters wider collaboration and increased citations
- maximum visibility for your research: over 100M website views per year
At BMC, research is always in progress.
Learn more biomedcentral.com/submissions

